Koumaré (2021)

\title{
ANSERJ
}

Vol. 12, No. 1

Spring / printemps 2021

pp. $69-81$

Canadian Journal of Nonprofit and Social Economy Research

Revue canadienne de recherche sur les OSBL et l'économie sociale

\section{Économie sociale et biens communs : cas de l'Association villageoise de Foabougou dans la zone de l'Office du Niger au Mali}

\author{
Mamadou Koumaré \\ Université des Sciences Sociales et de Gestion de Bamako, Mali
}

\begin{abstract}
This article deals with the production and management of collective goods and services by social economy organizations in Mali. Specifically, the article analyzes how a village association-a cooperative community organization-becomes involved in local socioeconomic development by pooling means and resources to produce self-managed collective goods and services for the benefit of its members, but also of non-members who have to pay more. This mode of organization allows rice producers to secure their living conditions in one of the largest hydro-agricultural enterprises in West Africa, as well as to participate in the development of their community.
\end{abstract}

\section{RÉSUMÉ}

Cet article aborde la question de la production et de la gestion de biens et services collectifs par les organisations de l'économie sociale au Mali. Précisément, l'article analyse la participation d'une association villageoise—une organisation communautaire à caractère coopératif-dans le développement socioéconomique local, par la mise en commun de moyens et de ressources afin de produire des biens et services collectifs autogérés au profit de ses membres, mais aussi de non-adhérents qui doivent payer plus. Ce mode d'organisation permet aux producteurs de riz de sécuriser leurs conditions de vie, dans l'une des entreprises hydro-agricoles les plus vastes de l'Afrique de l'Ouest, et aussi de participer au développement de leur communauté.

Keywords / Mots clés : Village association; Collective or common good; Social economy; Community management or self-management; Economic and social development / Association villageoise; Bien collectif ou commun; Économie sociale; Gestion communautaire ou autogestion; Développement économique et social

\section{INTRODUCTION}

L'Office du Niger est un vaste aménagement hydro-agricole créé au Mali en 1932 par la France. À cette époque, l'objectif était d'irriguer la vallée du fleuve Niger afin de produire du coton pour l'industrie française et du riz pour nourrir les travailleurs agricoles et plus tard les populations de l'Afrique occidentale française. De cette date à aujourd'hui, l'entreprise agricole a connu beaucoup de mutations, notamment dans les années 1980, à travers le Programme d'ajustement 


\section{Koumaré (2021)}

structurel (PAS). L'objectif du PAS à l'Office du Niger était le rétablissement de ses équilibres économiques et financiers. C'est dans ce contexte que l'Office s'est retiré des activités d'accompagnement, jugées non rentables, au profit des organisations paysannes comme les tons villageois, association de village ${ }^{1}$ et les sociétés coopératives créés à cet effet. Désormais, ces organisations sont responsables du crédit agricole, de l'accès des producteurs aux intrants, des opérations de battage, de décorticage et de commercialisation du riz et enfin du développement communautaire. Dès lors, nous assistons à un foisonnement d'organisations à caractère coopératif dans ladite zone, notamment des associations de village qui font l'objet de notre analyse. Dans ce cadre, nous avons choisi dans cet article de nous pencher sur le cas de l'association du village de Foabougou, lequel est situé dans la zone de l'Office du Niger, afin de voir comment ses membres mettent en commun leurs ressources pour produire et gérer des biens communs de façon autonome au profit de l'intérêt individuel et collectif.

L'article comporte quatre sections. La première pose la problématique de notre étude. Quant à la deuxième, elle apporte un éclaircissement sur les concepts clés de notre étude. La troisième section présente la démarche méthodologique, tandis que la quatrième présente les résultats de notre étude de cas sur le développement socioéconomique local. Enfin, nous discutons les résultats.

\section{PROBLÉMATIQUE}

L'Afrique a longtemps développé, à travers les organisations communautaires, des modes de production de biens et services communs fondés sur des solidarités socioculturelles ou ethniques au service de la reproduction économique, sociale et politique. En effet, les pratiques socioéconomiques africaines se caractérisaient par l'esprit communautaire et coopératif qui repose sur « des valeurs éthiques et morales, des consensus dans les échanges de travail, des systèmes de transmission des connaissances, des réflexes d'entraide et de défense » (Peemans, 2004). C'est dans ce contexte que certaines organisations communautaires comme les Naam ${ }^{2}$ au Burkina Faso, les samarya ${ }^{3}$ au Niger et les tons au Mali ont vu le jour pour donner sens au vivre ensemble par la production de biens et services communs.

En plus des organisations traditionnelles, nous avons assisté, à partir des années 80, à l'émergence d'autres formes d'organisation comme riposte à la précarisation des conditions de vie à la suite du PAS. ${ }^{4}$ En effet, cette période, marquée par une paupérisation des populations, a connu une véritable explosion d'organisations d'économie de type mutualiste, associatif et coopératif qui garantissent des fonctions de production économique, ainsi que de médiation sociale et politique (Assogba, 2000). Ces organisations sont chargées de répondre aux besoins des membres en matière de production, de commercialisation, de financement et d'accès aux services sociaux (logement, éducation, formation, santé).

Cependant, la place des pratiques socioéconomiques des producteurs locaux en Afrique, à travers des organisations sociales et solidaires, a longtemps été ignorée par beaucoup de chercheurs et spécialistes du développement. En effet, ces pratiques étaient considérées comme marginales car relevant beaucoup plus de la survie que d'une logique de développement (Lapeyre, 2006). Cependant, grâce à de nouveaux travaux de recherche, nous assistons depuis un temps en Afrique à une reconnaissance de ces pratiques socioéconomiques comme levier de développement local.

Ce nouveau regard sur les pratiques solidaires d'acteurs locaux comme champ d'étude et de développement continue de s'imposer comme une réalité incontournable (Peemans, 2005). Nous inscrivons notre recherche dans ce cadre à travers l'analyse des initiatives et pratiques d'entraide et de solidarité de production et de distribution de biens et services collectifs. En effet, à l'instar d'Andriamanindrisoa (2004), nous postulons que ces pratiques d'œuvrer ensemble, à travers des associations, relèvent d'une reproduction de type traditionnel, puisque les activités adoptées sont souvent celles que les aînés ou les grands-parents pratiquaient déjà. Elles s'inscrivent donc dans une forme de continuité, tout en s'adaptant aux mutations par l'acculturation, c'est-à-dire par l'incorporation et la modernisation. Elles concourent ainsi à 


\section{Koumaré (2021)}

travers « des réseaux de réciprocité et de redistribution » (Didier, 2010) à assurer la satisfaction des besoins socioéconomiques et sociopolitiques ignorés ou peu pris en compte par l'État et le marché.

Notre recherche se donne comme objectif de rendre compte des pratiques socioéconomiques d'acteurs locaux qui, comme le remarque Lapeyre (2006), sont loin d'être des « non-acteurs ». En effet, nous partons de l'idée que les « acteurs d'en bas » ne sont pas dépourvus de logiques de développement (Girard, 1994). Cela nous amène à formuler l'hypothèse selon laquelle les stratégies et pratiques associatives solidaires d'acteurs locaux pour la production de biens et services communs contribuent de manière significative au développement local. Celles-ci visent à mettre l'économique au service du développement local et du renforcement des liens sociaux et relèvent donc d'une logique de développement. Pour vérifier notre hypothèse, nous avons choisi d'étudier le cas de l'association villageoise de Foabougou. Ce choix s'explique par le fait que cette association est à la croisée des pratiques traditionnelles et modernes de production de biens communs en milieu rural. Elle nous offre donc la possibilité de questionner la place et le rôle des organisations traditionnelles à caractère coopératif dans le développement socioéconomique local. À travers ce choix, nous nous posons la question de savoir comment et en quoi cette association traditionnelle, confrontée à la modernité, contribue au développement de ce village—et au fait de toute la localité—à partir de la production de biens et services communs.

\section{CLARIFICATION DES CONCEPTS DE L’ÉCONOMIE SOCIALE, DE DÉVELOPPEMENT LOCAL ET DE BIEN COMMUN}

Dans cette section, nous définissons les perspectives conceptuelles et théoriques selon lesquelles nous envisageons les concepts clés de notre étude, c'est-à-dire l'économie sociale, le développement local et le bien commun.

Pour le concept de l'économie sociale, nous avons surtout exploité les travaux de Defourny (2006) qui ont l'avantage de mettre l'accent sur deux approches envers une définition de l'économie sociale, à savoir l'approche juridique et l'approche normative. L'approche juridique définit l'économie sociale selon les types de statut, soit la société coopérative, la mutuelle et les organisations associatives. Quant à l'approche normative, elle met en avant les valeurs et normes qui caractérisent l'économie sociale:

1. La finalité de service aux membres ou à la collectivité plutôt que de profit;

2. L'autonomie de gestion;

3. Le contrôle démocratique par les membres;

4. La primauté des personnes et de l'objet social sur le capital dans la répartition des excédents.

Pour notre étude, nous adhérons à l'approche normative (Defourny 2006). Ce positionnement théorique nous permet de rendre compte des réalités des pratiques des associations villageoises en matière de services aux membres ou à la collectivité et non la recherche de profit. En effet, l'une des caractéristiques de notre association villageoise est le service aux membres et à la communauté. En cela, elle répond directement à deux des principes de l'approche normative, à savoir la finalité de service aux membres ou à la collectivité plutôt que de profit et la primauté des personnes et de l'objet social sur le capital dans la répartition des excédents. En effet, les initiatives développées par l'association villageoise s'inscrivent dans le cadre de l'utilité de service aux membres et au développement de la localité. À cet effet, toutes les ressources générées sont utilisées de façon autonome et communautaire par ses membres qui ont les mêmes droits et la même voix dans la prise de décision.

À propos de l'utilité de service ou de finalité de services aux membres ou à la collectivité, les initiatives d'économie sociale et solidaire répondent généralement, selon Debuyst (2006), à des raisons d'être suivantes : 


\section{Koumaré (2021)}

1. L'inscription dans la mouvance historique d'une culture associative pour la défense et l'amélioration des conditions de vie des ouvriers, artisans, paysans et habitants des quartiers populaires urbains;

2. Les stratégies collectives de survie et de subsistance;

3. La réinsertion socioprofessionnelle;

4. La recherche d'une qualité de vie acceptable (services de proximité, conditions environnementales, aspects socioculturels);

5. L'insertion dans la gouvernance du développement local;

6. Les options citoyennes et d'opposition politique.

À travers ces objectifs, l'on se rend compte que l'économie sociale et solidaire participe pleinement au développement local. À ce propos, selon Démoustier (2002), les organisations de l'économie solidaire sont sollicitées pour mobiliser des acteurs collectifs, requalifier les groupes sociaux, mutualiser les ressources et répondre aux besoins et aspirations de la population. C'est ainsi que l'économie solidaire participe au développement local. En effet, l'auteur présente le développement local comme « un processus qui mobilise des personnes et des institutions cherchant à transformer l'économie et la société locales, en créant des opportunités de travail et de revenu afin d'améliorer les conditions de vie des populations ». De cette manière, on se trouve devant une initiative de développement local quand on constate l'utilisation de ressources et de valeurs locales sous le contrôle d'institutions et de personnes du niveau local, bénéficiant aux personnes et à l'environnement du territoire. Du rapport entre économie sociale et développement local, Fonda (2004) nous apprend que « les entreprises de l'économie sociale considèrent le territoire non comme simple opportunité, mais comme la base de leur activité et de leur développement. »

Pour en revenir aux objectifs de cet article, celui-ci se propose d'interroger la place des organisations de l'économie sociale et solidaire, notamment des associations villageoises, dans l'offre de services aux personnes et à l'environnement du territoire à partir de la gestion et de l'exploitation de biens communs. Dans cette perspective, nous définissons comme bien commun tout bien matériel ou immatériel relevant d'une appropriation commune ou « d'une propriété collective »donc d'un usage et d'une exploitation collectifs soumis à une gouvernance communautaire, par «l'émergence de formes d'action collective concertée » (Hardin, 1968). II s'agit donc de biens divisibles dont l'accès est communautairement contrôlé pour l'utilité aux membres sans que leur gestion ne soit soumise à une autorité institutionnelle spécifique. Compris dans ce sens, les biens communs selon Gadrey (2012) désignent des qualités de ressources ou un patrimoine collectif pour la vie et les activités humaines, ou des « qualités sociétales ». Ainsi, selon l'auteur, pour les qualifier de bien commun, il faut un jugement commun d'utilité collective selon lequel tous, par principe, devraient avoir la possibilité d'y accéder ou d'en bénéficier. Dans ce jugement, il y a de l'intérêt général, de l'utilité sociale ou sociétale, des « richesses collectives fondamentales ", des finalités ou valeurs de société, voire des droits universels. Vue sous cet angle, l'approche par les biens communs renvoie à la problématique de l'auto-organisation dont le capital social constitue le « liant » indispensable. Du coup, nous sommes bien au centre de la problématique de l'économie sociale et solidaire (ESS) car d'une manière plus générale, les entreprises de l'ESS, quel que soit leur statut, paraissent les plus à même de répondre à des demandes de gouvernance de biens communs venant de la société civile (Pérez et Silva, 2013).

\section{CADRE MÉTHODOLOGIQUE}

Notre démarche méthodologique se fonde sur les méthodes socio-anthropologiques que sont la recherche documentaire, la collecte de données sur le terrain et leur analyse. 


\section{La recherche documentaire}

Afin d'éclaircir les concepts d'économie sociale, de développement local et de bien commun, nous avons procédé à une exploitation de la littérature scientifique pour problématiser notre étude et définir notre perspective théorique par rapport à ces concepts.

Parallèlement à l'exploitation de la littérature grise, nous avons exploité différents rapports et outils de gestion que nous avons trouvés sur place auprès des sociétés coopératives et des associations. Cette exploitation nous a permis de disposer de données quantitatives et qualitatives sur les actions de développement initiées par les organisations, notamment les associations villageoises.

\section{La collecte de données sur le terrain}

Pour la collecte de données, nous avons utilisé l'entretien en groupe et l'entretien individuel. À cet effet, nous avons élaboré deux guides d'entretien adressés respectivement aux responsables des organes de gestion et aux membres de l'association. Dans le premier cas, nous avons discuté en entretien de groupe avec les membres des organes de gestion de l'association villageoise, soit en assemblée générale (3), soit en réunion de bureau (2). Les assemblées générales et les réunions de bureau ont l'avantage de réunir un grand nombre de membres, de permettre l'observation des dynamiques internes au groupe et de recouper les sources d'information. Dans le second cas, nous avons débattu individuellement (30), en fonction de leur disponibilité, avec quelques membres de l'association, de la chefferie locale et des groupements de jeunes et de femmes, soustraits de l'influence du groupe car il fallait recouper les sources d'information. Le tableau ci-dessous donne la situation des entretiens.

Tableau 1 : Nombre d'entretiens

\begin{tabular}{|l|l|c|}
\hline Types d'entretien & Cibles & $\begin{array}{c}\text { Nombre } \\
\text { d'entretiens }\end{array}$ \\
\hline \multirow{2}{*}{ Entretiens collectifs } & Membres de bureau & 3 \\
\cline { 2 - 3 } & $\begin{array}{l}\text { Membres de l'assemblée } \\
\text { générale }\end{array}$ & 2 \\
\hline \multirow{2}{*}{ Entretiens individuels } & Hommes & 20 \\
\cline { 2 - 3 } & Femmes & 10 \\
\hline Total & 35 \\
\hline
\end{tabular}

Dans l'ensemble, les entretiens se sont déroulés dans la langue bambara, parlée par plus de $95 \%$ de la population locale. Ensuite, nous avons traduit en français les données recueillies. Les entretiens ont porté sur des sujets tels que le fonctionnement de l'association, les modes de production et de gestion des biens et services communs, et leurs contributions au développement local.

\section{L'analyse des données}

Pour l'analyse des données, dans un premier temps, nous avons retranscrit les discours recueillis. Nous signalons que nos données sont qualitatives. Dans un deuxième temps, nous avons procédé à une analyse de contenu thématique afin d'étudier de façon systématique le rapport, l'interdépendance et l'interaction entre les pratiques développées par l'association ainsi que le développement local dans la zone de l'Office du Niger. 


\section{Koumaré (2021)}

\section{PRÉSENTATION DES RÉSULTATS}

Dans cette section de l'article, nous exposons les résultats de nos enquêtes de terrain. Nous mettons l'accent sur les activités de développement socioéconomique réalisées par l'association villageoise de Foabougou, d'abord au niveau du village, ensuite au niveau de la commune.

\section{L'association villageoise de Foabougou : mutualiser les ressources pour produire des services collectifs autogérés}

Organisation multifonctionnelle, cherchant en général à réunir tous les exploitants d'un même village, l'association villageoise continue d'être un outil indispensable de développement socioéconomique dans la zone de l'Office du Niger à travers des services multiformes autant individuels que collectifs. II est bon de rappeler que le contexte de création des associations villageoises dans la deuxième moitié des années 80 a été marqué par le début de la restructuration de l'Office du Niger, sous la houlette de la Banque mondiale. Par cette restructuration, l'entreprise agricole a vu ses missions recentrées. En effet, elle s'est retirée de certaines activités comme l'accès des paysans aux facteurs de production (équipements, crédits de campagne, intrants agricoles comme les semences et les engrais), la commercialisation des produits, et l'accès à des services sociaux de base. Afin de prendre désormais en charge ces différentes activités, les associations villageoises ont été créées ou ressuscitées (car elles font partie du patrimoine organisationnel local) dans chaque village de la zone. Ce rappel contextuel permet déjà de se faire une idée sur les orientations des différentes pratiques que développent ces associations.

En ce qui concerne concrètement l'association villageoise de Foabougou (notre étude de cas), elle a vu le jour en 1984, dans le cadre de la politique de développement local prônée par le régime d'alors, avec comme cellule de base les associations villageoises. À l'époque, dans la zone de l'Office du Niger, cette politique a bénéficié de l'appui des PaysBas qui ont doté les associations villageoises d'équipements collectifs de production et de commercialisation. C'est dans ce contexte que les habitants du village de Foabougou se sont organisés en association villageoise dont l'objectif était la promotion socioéconomique et sociopolitique de ses membres et du village. Cependant, l'association a connu à ses débuts des difficultés de fonctionnement, à la suite de problèmes de mauvaise gestion (détournements, non-maîtrise des outils de gestion) qui ont fortement divisé le village, d'autant plus que l'association du village d'à côté (2 km), à savoir Sériwala, était citée comme la référence de la zone et cette situation dérangeait un peu les habitants de Foabougou. Cette concurrence les poussa à se remettre en cause par la mise en place d'un mode de fonctionnement communautaire qui, même s'il n'était pas démocratique, accordait une grande place à la participation et à l'implication des composantes sociales. Par ce mode de fonctionnement amélioré, l'association villageoise de Foabougou constitue aujourd'hui l'une des meilleures associations villageoises engagées dans le développement villageois participatif.

L'association compte 250 ménages représentés par les chefs de ménage dont l'adhésion est libre et individuelle. Elle intervient selon son mandat dans le domaine de l'agriculture (riziculture et maraîchage) et du développement villageois.

\section{L'association villageoise de Foabougou fonctionne comme une organisation communautaire}

L'association villageoise de Foabougou dispose de trois organes de gouvernance, à savoir le comité de gestion, le conseil d'administration et l'assemblée générale. Pendant la période de campagne agricole, de mars à septembre, le comité de gestion se réunit tous les jeudis. Quant aux deux autres organes, ils se chevauchent dans leur fonctionnement. En effet, l'association villageoise de Foabougou fonctionne comme une organisation communautaire avec une importante place accordée au chef de village entouré de ses conseillers et aux composantes sociales du village (organisations des jeunes, de femmes et de religieuses). Dans les prises de décisions, bien qu'il ne soit pas membre du comité de gestion, le chef de village joue un rôle important en tant que garant moral. Ainsi, le comité de gestion, avant d'entreprendre toute action, soumet d'abord l'idée au chef de village qui, il faut le reconnaître, mène le jeu en s'assurant de l'adhésion et de la 


\section{Koumaré (2021)}

participation des habitants. Ensuite, le comité consulte les leaders des différentes composantes (notamment les jeunes et les femmes) ou organise une assemblée générale des gwatigis (chefs de ménage). Lors des rencontres des membres, le mode consensuel s'impose quant à la prise de décisions. La recherche du consensus vise à éviter tout clivage qu'entraînerait un vote. C'est pourquoi, en cas de blocage, le chef de village inclut son réseau de conseillers dans les négociations. Cette démarche permet d'obtenir des compromis.

Une autre pratique permettant à l'association villageoise de Foabougou d'impliquer ou de responsabiliser les membres est la multiplication de petits groupes de travail chargés de domaines spécifiques comme la gestion de l'école communautaire ou celle du problème de la santé (case de santé). Malgré ce dispositif, nous relevons quelques tensions au niveau de l'utilisation des ressources où certains membres reprochent au comité de gestion de ne pas se consacrer suffisamment aux problèmes récurrents de crédits de campagne et de commercialisation ou de ne pas suffisamment les prendre en compte dans l'évaluation des besoins en intrants.

\section{L'animation économique au sein du village repose essentiellement sur l'association villageoise}

Sur le plan économique, l'association développe plusieurs pratiques au profit de ses membres et de toute la communauté.

L'accès collectif aux crédits de campagne et aux intrants à travers l'association villageoise

Au niveau de l'association villageoise de Foabougou, l'accès aux facteurs de production agricole concerne tous les habitants du village. Cet accès constitue d'ailleurs sa force. À cet effet, un comité a été mis en place pour l'identification et l'estimation des besoins en intrants agricoles et crédits de campagne. D'abord, ce comité contacte tous les chefs d'exploitation résidant dans le village. Ensuite, il convoque une assemblée générale de validation qui décide de l'option par rapport à l'organisation de la campagne à venir : octroyer directement les financements aux exploitants ou acheter des intrants à répartir entre les membres. Après une première expérience difficile d'allocation de fonds aux exploitants, à la suite de leur utilisation à d'autres fins, l'association villageoise a décidé, il y a plus d'une décennie, d'acheter ellemême les intrants et de les partager entre les membres. Cette option, même si elle exige plus de rigueur dans la gestion quand on sait que les responsables de l'association ont des difficultés à gérer les documents de gestion, a l'avantage de diminuer le coût de cession des intrants. En effet, plus la commande est groupée (donc importante), plus le prix de l'intrant baisse. Aussi, selon le secrétaire général du comité de gestion, « Le fait de procéder à l'achat collectif pour le village leur permet de bénéficier de crédits à des taux réduits. En effet, plus le montant des crédits est élevé, plus bas est son taux d'intérêt. » Depuis quelques années, la Banque nationale de développement agricole (BNDA) accorde des prêts à l'association à un taux de $7 \%$, ce qui est raisonnable quand on sait que le taux d'intérêt peut aller jusqu'à $25 \%$ auprès des institutions de microfinancement qui préfèrent les prêts de courte durée (de 4 à 8 mois).

Quant au remboursement de ces intrants, il se fait en espèces. Ainsi, à la fin de la campagne, l'exploitant paie en liquide le prix du nombre de sacs reçus, plus les intérêts de la BNDA et quelques frais de manutention. Une commission de recouvrement est mise en place à cet effet. En cas de difficulté de remboursement, l'association villageoise paie à la place du débiteur qui n'aura désormais accès à ses services qu'après s'être acquitté de sa dette avec pénalité.

Nous relevons que cette pratique est d'une importance capitale dans l'amélioration de la productivité et de la diminution des coûts de production. En effet, l'une des principales difficultés auxquelles les paysans sont confrontés est l'accès à temps aux intrants qui affichent parfois des prix élevés ou manquent sur le marché. La période de précampagne est assez délicate pour les exploitants car à ce moment beaucoup d'entre eux connaissent une difficulté de trésorerie et ne sont pas en mesure d'accéder aux intrants sans l'appui de l'association villageoise. Ainsi, celle de Foabougou, par sa solidité financière, notamment sa capacité à prendre en charge des frais supplémentaires (par exemple, la manutention), 


\section{Koumaré (2021)}

parvient à faire diminuer le prix de cession des intrants. En effet, selon le président, l'association utilise ses propres ressources et moyens pour la mobilisation des crédits, mais aussi pour l'achat et l'acheminement des intrants jusqu'aux magasins de stockage de l'association dans le village. Le fait que l'association utilise ses propres moyens de transport réduit les frais liés au transport car elle n'achète que le carburant. Aussi, le fait d'utiliser ses propres ressources financières la met à l'abri du prêt bancaire.

Des infrastructures et équipements collectifs pour réduire les charges de production et faciliter la commercialisation Foabougou est l'un des villages les plus dotés en infrastructures de stockage. Celles-ci ont été construites par l'association villageoise. Depuis son lancement en 1984, avec l'appui de l'organisme néerlandais qui a financé les deux premiers magasins en banco, l'association villageoise de Foabougou a pu réaliser elle-même sur fonds propres deux autres magasins en ciment. En effet, les équipements collectifs de l'association lui ont permis de générer d'importantes recettes au moyen de différentes prestations de services marchands.

II est utile de signaler aussi que les magasins servent à stocker les récoltes des paysans en riz paddy (riz non décortiqué) ainsi qu'en riz décortiqué. En effet, d'une manière générale, les exploitants de la zone de l'Office du Niger sont confrontés au manque d'infrastructures appropriées pour le stockage car celles construites dans les années 80 avec l'appui des Pays-Bas se sont écroulées ou ne sont plus en bon état parce que faites en banco. Cette situation a conduit l'association villageoise de Foabougou à désormais construire en ciment.

Pour l'exploitation des magasins, à la fin de la récolte, les paysans confient au gérant du magasin leurs récoltes en riz paddy ou décortiqué sur la base de documents écrits. Les magasins sont dotés de deux clés dont l'une est détenue par le président et l'autre par le gérant. Pour l'association villageoise de Foabougou, ce service est gratuit. II lui permet de rassembler sur place d'importantes quantités de la production du village pour le battage, le décorticage et la commercialisation collective. En effet, au niveau des magasins, tous les équipements intervenant dans ces opérations (bascule, batteuse, décortiqueuse) sont déjà sur place, ce qui réduit les coûts, comme nous l'avons signalé.

En ce qui a trait à la mise en place et l'exploitation des équipements collectifs, l'association villageoise de Foabougou dispose de quatre batteuses et de trois décortiqueuses de riz acquises sur fonds propres. Ces équipements ont été obtenus à partir de l'exploitation de deux premières batteuses acquises sur financement néerlandais et sur prêt pour deux décortiqueuses. Pour leur exploitation, en fonction de la réussite de la campagne, l'assemblée générale des membres fixe les coûts des différents services à rendre aux membres et à d'autres exploitants. II s'agit principalement du décorticage et du battage. Par rapport au battage, pour les membres, sur dix sacs de riz paddy, un revient à l'association villageoise. Pour un non-adhérent, l'association retient deux sacs sur dix. Concernant le décorticage, la prestation se paie en général au comptant, soit $1500 \mathrm{~F}$ par sac de $100 \mathrm{~kg}$ pour les membres contre $2000 \mathrm{~F}$ pour les non-adhérents.

Pour la réussite des opérations de battage et de décorticage, l'association villageoise a engagé cinq ouvriers saisonniers payés journalièrement. L'utilisation des équipements se fait sur demande des membres qui assistent à tout le processus, au terme duquel un document faisant le point par membre est établi. Signalons que ces équipements fonctionnent avec du gasoil, ce qui engendre des dépenses et des frais d'entretien ou de réparation. Ces éléments, particulièrement le prix du carburant, entrent en ligne de compte dans la fixation des prix de location des machines. Les recettes issues de l'ensemble des opérations sont gardées par le trésorier qui tient un compte bancaire avec le président de l'association villageoise. Ces recettes, comme nous le verrons ci-dessous, sont utilisées pour le développement villageois-notamment, l'accès aux services sociaux de base. 


\section{Koumaré (2021)}

Les produits sont vendus collectivement et l'association villageoise négocie elle-même avec les commerçants grossistes

Concernant la commercialisation, la pratique de l'association villageoise de Foabougou consiste à la vente collective des récoltes une fois que le cours du riz a atteint un prix jugé acceptable par les paysans. Pour cette vente, l'association villageoise ne passe pas par une structure intermédiaire spécialisée dans la vente du riz. Elle démarche elle-même les commerçants grossistes. Pour que les producteurs s'en sortent bien, le prix au kilogramme doit atteindre au moins $300 \mathrm{~F}$ CFA. Ce montant est difficile à atteindre malgré la bonne qualité gustative (appréciée dans la sous-région) du riz de l'Office car le marché malien est envahi par le riz thaïlandais de prix inférieur et de qualité moindre. Le fait que les producteurs ne peuvent pas garder très longtemps leurs productions explique aussi leur difficulté à obtenir un montant raisonnable, bien que l'association achète parfois à ses membres en difficulté.

\section{LE DÉVELOPPEMENT DES ACTIVITÉS ÉCONOMIQUES PERMET L'AMÉLIORATION DE L'ACCĖS COLLECTIF AUX SERVICES SOCIAUX}

Nous relevons que l'une des forces de l'association villageoise de Foabougou tient de sa capacité à lier développement économique et social. Nous l'avons déjà indiqué, le reproche qui se fait le plus souvent aux associations villageoises est de privilégier la dimension sociale du développement aux dépens de la dimension économique. En effet, en général, les associations villageoises consacrent l'essentiel de leurs ressources financières au financement des activités sociales au point d'épuiser ces ressources. Du coup, ces associations, n'arrivant plus à assurer des services aux membres, sont obligées de mettre la clé sous la porte. L'association villageoise de Foabougou a compris que l'un ne peut aller sans l'autre. En effet, sans une solidité financière, il est impossible de faire des prestations sociales dont la satisfaction motive les membres de l'association à s'engager davantage dans la vie de leur organisation.

À ce propos, le président de l'association de Foabougou affirme,

Nous tenons à l'équilibre de notre association en ne faisant pas de prestations sociales qui videraient notre caisse comme c'est le cas pour d'autres associations. Nous avons été confrontés à ce problème au début. Désormais les prestations sociales dépendent de la situation de la caisse.

Cette démarche de l'association, pouvant d'un côté être vue comme une forme de retrait et de désengagement, a pourtant l'avantage, dans une optique d'hybridation entre logique sociale et logique économique, de permettre la pérennité et la durabilité de ses actions. Cela explique, entre autres, sa longévité (rappelons qu'elle a été créée en 1984).

En termes de pratiques de développement sur le plan social, nous notons surtout celles consistant à mettre en place des écoles communautaires et des centres d'alphabétisation. En effet, l'accès aux services éducatifs passe par ceux-ci. L'association villageoise de Foabougou joue dans ce domaine le rôle de collectivité publique car presque tous les services offerts sont à mettre à son compte.

Concernant la scolarisation des enfants, l'organisation villageoise a établi une école communautaire de premier cycle de six classes (dont trois en banco et trois autres en ciment). Cette école compte en moyenne 300 élèves et est dirigée par un comité de gestion mis en place par l'association villageoise. Ce comité est chargé d'assurer le fonctionnement de l'école au jour le jour (prise en charge du salaire des enseignants, achat de la craie et d'autres consommables), mais aussi de la sensibilisation des parents pour la scolarisation des enfants. Pour assurer le fonctionnement, le comité a instauré une cotisation mensuelle de 1000 F CFA par enfant. Depuis 2011, l'école communautaire a été érigée en école publique par le conseil communal qui participe désormais à la prise en charge des salaires des enseignants. 


\section{Koumaré (2021)}

Afin de permettre aux enfants du village de continuer leurs études au second cycle, l'association villageoise de Foabougou et celle du village de Sériwala (à $2 \mathrm{~km}$ ) ont conjugué leurs efforts pour construire à Sériwala une école de second cycle qui elle aussi est devenue publique, en 2011. Sur ce sujet, le président du comité de gestion nous rapporte, "Compte tenu du fait que l'association n'a pas les moyens d'assurer à elle seule la construction et le fonctionnement d'une école de second cycle, nous avons décidé de fédérer nos moyens avec ceux de l'association du village de Sériwala qui est confrontée au [même] problème. »

Pour la formation des adultes, l'association villageoise a mis en place un centre d'alphabétisation dans les années 90. Cependant, ce centre ne parvient pas à organiser régulièrement des sessions de formation faute d'auditeurs, notamment chez les hommes qui sont moins assidus que les femmes car celles-ci semblent s'intéresser plus à cette formation. L'association estime le nombre d'adultes alphabétisés à seulement 320 personnes dans le village sur un total de 3936 habitants.

Au vu de ces réalisations, nous osons dire que l'association villageoise de Foabougou remplace pratiquement le conseil communal pour ce qui est de l'accès des habitants aux services de l'éducation. Nous constatons qu'en dehors de la prise en charge d'une partie des salaires des enseignants, le conseil communal a pendant longtemps été pratiquement absent dans la production de services éducatifs même si, ces dernières années, il commence à prendre en charge le fonctionnement des écoles communautaires. II n'empêche que l'association villageoise, grâce à la mutualisation des moyens, reste le principal acteur dans l'accès à ce service qui est public mais qui perdure grâce à une gestion communautaire.

\section{DISCUSSION DES RÉSULTATS}

À travers tout ce qui précède, nous voyons que l'association villageoise dans la zone de l'Office du Niger constitue un acteur majeur de développement touchant à la fois les dimensions économique et sociale. Dans cette entreprise hydroagricole nationale qu'est l'Office du Niger, les paysans doivent faire face à la mondialisation et à un programme d'ajustement structurel qui a vu l'État se retirer des activités d'accompagnement jugées non rentables. Néanmoins, en dépit de leur précarisation, ces paysans réussissent à développer de plus en plus de stratégies de mutualisation de leurs ressources. Ils ont compris qu'ils doivent s'organiser de façon solidaire pour assumer leur propre développement, ce qui explique le foisonnement d'organisations de l'économie sociale et solidaire dans la zone de l'Office du Niger.

Notre étude de cas nous a permis de mettre en évidence comment l'association villageoise participe à la réalisation du développement local par la mise en place d'équipements et de services collectifs, pour atteindre une forme d'organisation communautaire à caractère coopératif mais sans fondement juridique. En effet, nous avons relevé que la quasi-totalité des réalisations économiques et sociales effectuées dans les différents villages sont pratiquement à l'actif de leurs associations villageoises. Malgré l'avènement des collectivités territoriales dans les années 90 , notamment les communes, les organisations associatives continuent d'être un acteur majeur du développement socioéconomique local.

Ce constat s'impose particulièrement dans le domaine économique, où depuis le recentrage des missions de l'Office du Niger, ce sont les organisations associatives villageoises qui ont pris la relève et développent de façon continue des pratiques animant l'économie de la zone. À défaut d'une étude des impacts de ces pratiques, nous pouvons affirmer que les associations villageoises, qui deviennent de plus en plus de véritables sociétés coopératives, sont de véritables outils de développement économique dans la zone de l'Office du Niger. En effet, aujourd'hui, elles assurent l'accès du plus grand nombre de paysans aux facteurs de production, ainsi que la mise en place d'équipements et d'infrastructures de production, de stockage et de commercialisation des produits, non seulement pour leurs membres mais aussi pour les communautés villageoises entières. Ces pratiques concourent à améliorer les revenus des exploitants et par conséquent leur condition de vie. La question est de savoir si elles le font suffisamment et si elles peuvent s'inscrire 


\section{Koumaré (2021)}

dans la pérennité, quand on sait que la majorité d'entre elles sont confrontées parfois à des problèmes de gouvernance (mauvais choix des dirigeants, mauvaise gestion des ressources, pilotage à vue, etc.) et de manque de ressources qui limitent considérablement leur efficacité. Celle-ci est tellement importante vu que la durabilité et la dimension des actions de développement local dépendent des capacités d'autofinancement des organisations; elles doivent pouvoir compter sur leurs propres ressources, en plus d'avoir la capacité de gérer des ressources importantes. Les paysans doivent désormais faire preuve de plus d'innovation pour continuer à assurer plus de services au plus grand nombre de membres à partir de principes de solidarité et de mutualisation face aux difficultés de financement des activités agricoles. Un autre défi à relever consiste à œuvrer avec d'autres organisations à caractère coopératif et à saisir les conjonctures favorables en s'ouvrant à d'autres acteurs. En effet, il est important pour ces organisations d'avoir un ancrage local et identitaire, mais elles doivent s'adapter à l'évolution de l'environnement institutionnel et organisationnel pour changer de dimension. La capacité des associations villageoises à mobiliser plus de ressources et à développer des pratiques durables plus visibles dépend de leur mode d'organisation et de leur arrimage avec l'extérieur.

Malgré ces insuffisances, nous pouvons dire que notre hypothèse de recherche a été confirmée, car les résultats montrent bien l'important rôle de l'association villageoise dans le développement de la localité. À ce titre, notre recherche rejoint les conclusions d'autres travaux de recherche sur la question. En effet, à propos de l'importance des associations villageoises dans le développement local, Jacop et Delville concluaient que les organisations et groupements en milieu rural africain se réclament du développement; ceux-ci cherchent à améliorer l'équipement des villages, à mettre en place des services sociaux, et à établir des activités productives. Pour les deux auteurs, le fait « de décider collectivement de construire des infrastructures d'intérêt général, de créer des services socio-économiques (banque de céréales, moulin à mil, etc.) témoigne bien d'un projet, au moins implicite, sur le devenir du village, d'une prise en charge, à travers l'équipement, des "affaires publiques locales" » (Jacop et Delville, 1994, p. 11). Dans la même dynamique, Lapeyre (2006) affirme que « les acteurs locaux ne sont en rien passifs mais au contraire activement engagés dans la définition permanente de leur rapport à la nature et de leur mode de vie, afin de sécuriser leurs conditions de vie sur un territoire ». Aussi, pour Andriamanindrisoa (2004), les acteurs locaux développent des pratiques de formalisation et d'incorporation dans la logique des pratiques de vie relativement autonomes; on ne peut pas simplement réduire ces acteurs au rang de pauvres cherchant des offrandes provenant d'ambitieux programmes de lutte contre la pauvreté. Enfin, Parodi (2005) nous apprend que le projet collectif et le plus souvent le développement local sont au cœur même de l'émergence de toute expérience d'économie sociale et solidaire, car chacune de ces expériences contribue en retour à façonner des territoires dans le cadre de proximités aux déclinaisons multiples. La question qui se pose est la reproductibilité dans le temps et dans l'espace de ces expériences et leur contribution durable au développement local et plus largement à celui d'un patrimoine territorial.

\section{CONCLUSION}

L'étude des pratiques de production de biens et services communs par l'association du village de Foabougou nous a révélé que les organisations à caractère coopératif de la zone de l'Office du Niger participent à la réalisation du développement local par la mise en place de sources de financement (crédits de campagne), d'intrants agricoles (notamment les engrais), d'équipements collectifs de production, d'infrastructures collectives de stockage et de conservation, et ce au profit de leurs membres et de la communauté entière. En outre, ces mêmes organisations participent à la mise en place de services sociaux, car elles sont construites autour de valeurs d'entraide, de solidarité et de réciprocité qui tentent de combiner utilité sociale et économique.

Sur le plan économique, nous avons démontré que ces pratiques cherchent à lier la croissance des activités à la valorisation des personnes et des communautés : l'amélioration des revenus, le renforcement des capacités, la création et le maintien de l'emploi. Nous avons donc compris que ces pratiques occupent un important rôle dans l'amélioration 


\section{Koumaré (2021)}

des conditions de vie des paysans et dans le développement du territoire sur lequel ils vivent. Sur le plan social, nous avons retenu que tout en participant à la construction de réseaux de sociabilité, l'association villageoise a développé des pratiques qui ont servi à mettre en place des services sociaux spécifiques (écoles communautaires, centres de santé, etc.). Elle compense de ce fait l'offre de services insuffisante de l'État dans les secteurs de l'éducation et de la santé.

D'une manière générale, l'analyse de ces résultats nous a démontré que l'animation socioéconomique au sein des villages repose essentiellement sur les associations villageoises. En effet, même si ces organisations sont confrontées à des problèmes de coordination et parfois de faiblesse de moyens financiers, elles parviennent à rendre des services socioéconomiques individuels et collectifs à leurs membres, voire à toute la communauté, en lieu et place de l'État qui s'est retiré des services jugés non rentables à la suite de la crise.

L'analyse nous a révélé aussi que plus les associations rendent de services à leurs membres ou aux collectivités, plus elles garantissent les conditions d'une grande participation et implication de l'ensemble des acteurs locaux, facteurs nécessaires à la réussite et au développement durable d'initiatives et d'innovations socioéconomiques locales. Du coup, cela confirme notre hypothèse qui affirmait que les stratégies et les pratiques solidaires d'acteurs locaux, à travers les associations, notamment villageoises, participent au développement local. À ce titre, nous avons trouvé que notre recherche rejoint les conclusions d'autres travaux de recherche sur la question (Andriamanindrisoa, 2004; Lapeyre, 2006; Parodi, 2005). Ainsi, nous estimons pouvoir donner notre contribution au débat sur la reconsidération et la relecture des potentialités de développement local des associations à caractère coopératif séculaires africaines agissant à travers la production de biens et services communs.

\section{NOTES}

1. Le ton villageois et les associations villageoises sont des organisations communautaires et séculaires qui se définissent comme des instruments d'action et de promotion de la communauté villageoise. Ils constituent la structure opérationnelle locale qui gère le développement économique, social et culturel du terroir villageois dans le cadre du développement local. Ils s'identifient aux intérêts collectifs et individuels de ses membres et reposent sur l'engagement volontaire de chacun d'eux dans la planification, la prise de décision, l'exécution et le contrôle de ses activités.

2. Le groupement Naam est une association traditionnelle communautaire au Burkina Faso. II regroupe les jeunes d'un même village ou d'un même quartier autour d'activités agricoles, sociales et culturelles. Cette forme d'organisation traditionnelle a été réhabilitée dans les années 60 pour regrouper les paysans dans des organisations coopératives.

3. La samarya est une organisation traditionnelle d'entraide et de solidarité pour les jeunes en milieu rural au Niger. Elle s'occupe des questions de développement communautaire. Dans les années 70 , cette forme d'organisation a servi de base pour la mise en place d'une politique de développement rural dont l'un des objectifs était d'organiser les paysans pour leur meilleur encadrement.

4. Le Programme d'ajustement structurel (PAS) a exigé certaines conditions aux pays, notamment la déflation, c'est-àdire la diminution de l'effectif des fonctionnaires de l'État. À cet effet, l'État devait fermer certains services de santé et d'éducation. Aussi, pour les fonctionnaires, il devait mettre en place une politique de départ volontaire à la retraite.

\section{RÉFÉRENCES}

Andriamanindrisoa, E-A. (2004). Économie populaire, territoires et développement à Madagascar : les dimensions historiques, économiques et socioculturelles du Fokonolona. Études de cas : la commune rurale de Masindray et la commune urbaine d'Anosibe (thèse de doctorat). Louvain-la-Neuve, BE : Université Catholique de Louvain.

Assogba, Y. (2000). Gouvernance, économie sociale et développement durable en Afrique. Cahiers de la Chaire de recherche en développement communautaire (CRDDC). Série Recherche, 16. Hull, QC : Université du Québec à Hull, 1-30. 


\section{Koumaré (2021)}

Battesti, R. et al. (2005). Économie sociale et solidaire, territoires et proximité. Revue internationale de l'économie sociale, 296, 8-25.

Dacheux, E. et al. (2011). Principes d'économie solidaire. Paris, FR : Ellipses.

Debuyst, F. (2006). Limites et complémentarité d'une socio-économie associative. Dans C. Auroi et al., Économie solidaire et commerce équitable : acteurs et actrices d'Europe et d'Amérique latine. UCL, Presses universitaires de Louvain, IUED.

Defourny, J. et al. (2006). L'économie sociale du Nord au Sud, $2^{\mathrm{e}}$ édition. Bruxelles, BE : De Boeck Université.

Didier, K-K. (2010). Territoire produit par l'industrie minière et rupture de filiation ouvrière. Dynamique populaire et territoriale dans les cités minières de la Gécamines $(R D C)$ (thèse de doctorat). Louvain-la-Neuve, BE : Université Catholique de Louvain.

Eme, B. et al. (2006). Économie solidaire. Dans J.-L. Laville et al., Dictionnaire de l'autre économie. Paris, FR : Desclée de Brouwer.

Gadrey, J. (2012). Des biens publics aux biens communs (blog). Alternatives économiques, 24 avril.

Girard, C. (1994). Politiques publics de coopérations au développement et organisations paysannes africaines : l'exemple des communautés européennes. Dans J.-P. Jacop et Ph.-L. Delville, Les associations paysannes en Afrique : organisation et dynamiques. APAD - Karthala - IUED. Paris, FRz; édition Karthala.

Hardin, G. (1968). The Tragedy of the Commons. Science, New Series, 162(3859), pp. 1243-1248.

Jacop, J.-P. et P.-L. Delville. (1994). Les associations paysannes en Afrique : organisation et dynamiques. Paris, FR : APAD-Karthala-IUED.

Lapeyre, F. (2006). Mondialisation, néo-modernisation et « devenir » : un autre regard sur les pratiques populaires. Dans G. Froger, La mondialisation contre le développement durable? Paris, PIE Peter Lang, collection Ecopolis.

Lascoumes, P. et Jean-Pierre, L.-B. (1998). Le bien commun comme construit territorial. Identités d'action et procédures. Politix, 42, 37-66.

Lascoumes, P. et al. (2010). Instrument. Dans L. Boussaguet et al., Dictionnaire des politiques publiques, $3^{e}$ édition. Paris, FR : Presses de Sciences Po.

Laville, J.-L. (2000). L'économie sociale et solidaire en Europe. Les notes de l'Institut Karl Polanyi. Impatiences démocratiques éditeur.

Marthe, N., et al. (2000). Une solidarité en actes. Gouvernance locale, économie sociale, pratiques populaires face à la globalisation. Louvain-la-Neuve : UCL/Presse Universitaires de Louvain.

Mertens, S. (2001). Clarification conceptuelle : vers un consensus sur l'usage des termes. Dans Université de Liège et al., Économie sociale : enjeux conceptuels, insertion par le travail et service de proximité. Bruxelles, BE : De Boeck Université.

Parodi, M. (2005). Économie sociale et solidaire et développement local. Revue internationale de l'économie sociale, 296, 26-41.

Peemans, J.-P. (2004). Quelques remarques introductives. Dans Sophie Charlier et al., (2017), Articuler communs et économie solidaire : une question de gouvernance? RECMA, 345(3), pp. 27-41.

Pérez, R., et Silva, R. (2013). Gestion des biens collectifs, capital social et auto-organisation : l'apport d'Elinor OSTROM à l'économie sociale et solidaire. Management \& Avenir, 65(7), 2013, pp. 94-107.

Ricardo, P. (1996). Le bien commun : éloge de la solidarité. Cahiers libres, éd. P 2, Quotidien Le courrier, Labor. Singer, P. (2006). Économie solidaire. Dans J.-L. Laville et al., Dictionnaire de l'autre économie. Paris, FR : Desclée de Brouwer.

\section{L'AUTEUR / ABOUT THE AUTHOR}

Mamadou Koumaré est enseignant à l'Université des Sciences Sociales et de Gestion de Bamako (USSGB), Mali. II est détenteur d'un doctorat en études/sociologie de développement. Adresse : Rectorat de l'USSGB au Quartier du Fleuve, Bamako, Rue 310, Porte 238, BP : 2575. Courriel : koumaresdses@hotmail.fr 